\title{
The Maillard protein cross-link pentosidine in urine from diabetic patients
}

\author{
M. Takahashi ${ }^{1}$, T. Ohishi ${ }^{1}$, H. Aoshima ${ }^{1}$, K. Kawana ${ }^{1}$, K. Kushida ${ }^{1}$, T. Inoue ${ }^{1}$, K. Horiuchi ${ }^{2}$ \\ ${ }^{1}$ Department of Orthopaedic Surgery, Hamamatsu University School of Medicine, Hamamatsu, Shizuoka, Japan \\ ${ }^{2}$ Department of Chemistry, Hamamatsu University School of Medicine, Hamamatsu, Shizuoka, Japan
}

Summary. The Maillard protein cross-link pentosidine is a fluorescent condensation product of lysine, arginine and ribose. It accumulates in human tissues with age, and the accumulation process is accelerated in the tissues of diabetic patients. Using SP-Sephadex C-25 in the pretreatment for HPLC, we examined levels of pentosidine in urine without hydrolysis (free form) and levels of pentosidine in urine after hydrolysis (total forms), from 23 diabetic patients and 21 control subjects. The mean percentages of the values of free form per total forms $( \pm S D)$ were $89 \pm 15 \%$ in diabetic patients, $88 \pm 16 \%$ in control subjects and $89 \pm 15 \%$ in total populations of diabetic patients and control subjects. There was a significant correlation between the values of free form and total forms in diabetic patients $(r=0.938, p=0.0001)$, in con- trol subjects $(r=0.820, p<0.02)$ and in total populations of diabetic patients and control subjects $(r=0.951, p=0.0001)$. The mean level of pentosidine per mol creatinine ( \pm SD) was significantly elevated in urine from diabetic patients as compared to the level in control subjects $(8.8 \pm 4.3 \mu \mathrm{mol} / \mathrm{mol}$ creatinine vs $4.2 \pm 1.4 \mu \mathrm{mol} / \mathrm{mol}$ creatinine, $p=0.0001$ in free form; $10.1 \pm 5.3 \mu \mathrm{mol} / \mathrm{mol}$ creatinine vs $4.7 \pm 1.4 \mu \mathrm{mol} / \mathrm{mol}$ creatinine, $p=0.0001$ in total forms). These results demonstrate that urinary pentosidine, especially in free form, could be a useful marker for the assessment of diabetes and diabetic complications.

Key words: Fluorometry, HPLC, human urine, laboratory medicine.
Proteins in long-lived tissues are known to be modified by reducing sugars post-transcriptionally $[1,2]$. Of these compounds, Amadori products of the sugars, especially glycated haemoglobin, are popular for use in the clinical treatment of patients with diabetes mellitus [3]. These unstable compounds undergo many reactions progressively in vivo, and result in advanced Maillard or glycation end products $[1,2]$. Such modifications accelerate in their formation under diabetic conditions. Some stable end products such as $\mathrm{N}^{\mathrm{E}}$-(carboxymethyl)hydroxylysine, $\mathrm{N}^{\mathrm{E}}$-(carboxymethyl)lysine and fructoselysine were studied to determine the effects of diabetic conditions on their formation in humans $[2,4-6]$. However, since the detection of these end products is complicated, the method may be unsuitable for clinical use.

Pentosidine, characterized by Sell and Monnier [7] in human dura mater collagen, is a bifunctional condensation product of arginine, lysine and ribose, and is a fluorescent compound. Recent studies have revealed that its formation in human tissues is affected by the diabetic condition $[1,2,4,5,8-10]$. Until recently, only one study measuring the levels of pentosidine in body fluids (i. e. pentosidine levels in hydrolysate of human plasma) has been reported by Odetti et al. [8]. Since their method required the combined reverse-phase ion-exchange HPLC, a new and simple method needs to be developed to analyse levels of pentosidine in body fluids as a marker for the assessment of diabetes and diabetic complications. In a previous study, we developed a method to measure pentosidine in hydrolysates of human urine using SP-Sephadex C-25 in the pretreatment for HPLC, and discovered that urinary pentosidine increased exponentially with age in normal subjects [11]. In this study, we applied this method to examine whether pentosidine in urine could be a biomarker in the management of patients with diabetes.

\section{Subjects and methods}

\section{Subjects}

Diabetic patients ( $n=23,13$ males and 10 females) aged 46 to 75 years (mean $\pm S D, 62.2 \pm 8.6$ years) were randomly selected following routine visits to the Division of Internal Medicine at the Hamamatsu National Hospital in Hamamatsu, Shizuoka. All the patients had Type 2 (non-insulin-dependent) diabetes currently being treated with insulin or oral hypoglycaemic agents. Any 
patient who showed renal dysfunction was excluded from the study. As an age-matched control group, urine was collected from 21 healthy volunteers ( 9 males and 12 females), aged 49 to 73 years (58.6 \pm 6.9 years) with no history of diabetes and no current known disease. Urine samples were collected in the morning between 09.00 hours and 11.00 hours and stored at $-30^{\circ} \mathrm{C}$ until analysis.

\section{Reagents}

The following reagents were obtained from Wako Pure Chemical Industries (Osaka, Japan), HPLC-grade acetonitrile (MeCN), sequenation-grade n-heptafluorobutyric acid (HFBA), reagent-grade $\mathrm{HCl}$. All water used in these experiments was purified using a MilliQ, Water Purification System (Millipore Corporation, Bedford, Mass. USA).

\section{Prefractionation of urine or its hydrolysates with SP-Sephadex C-25}

We identified the pentosidine in urine without hydrolysis as the free form of pentosidine, and that in a hydrolysate of urine as the total forms of pentosidine. After thawing, a 2-ml aliquot of urine was hydrolysed with an equal volume of $12 \mathrm{~mol} / 1 \mathrm{HCl}$ at $110^{\circ} \mathrm{C}$ for $20 \mathrm{~h}$ in a sealed glass tube. Urine $(0.125 \mathrm{ml})$ or its hydrolysate $(0.25 \mathrm{ml})$ was mixed with $15 \mathrm{ml}$ of water and applied to an SP-Sephadex C-25 column $\left(\mathrm{H}^{+}\right.$form, $0.8 \times 1.0 \mathrm{~cm}$; Pharmacia LKB Biotechnology AB, Uppsala, Sweden) that had been equilibrated with water. The column was washed with $20 \mathrm{ml}$ of $0.1 \mathrm{~mol} / 1 \mathrm{HCl}$ and pentosidine was eluted with $5 \mathrm{ml}$ of $1.0 \mathrm{~mol} / 1 \mathrm{HCl}$. The eluate was evaporated under vacuum, and the residue was dissolved in $200 \mu \mathrm{l}$ of $1 \%$ HFBA. The solutions were stored at $-30^{\circ} \mathrm{C}$ prior to the HPLC analysis.

\section{High-performance liquid chromatography}

The HPLC system consisted of a Model CCPM pump (Tosoh, Tokyo, Japan), a Model FS-8010 spectrofluorometer (Tosoh), a Model AS-8010 autosampler (Tosoh), and a Model SC-8010 system controller (Tosoh). A column $(8 \mathrm{~mm} \times 10 \mathrm{~cm})$ prepacked with Radial-Pak C18, of 10- $\mu \mathrm{m}$ particle size, type $8 \mathrm{C1} 1810 \mu$ (Waters Associates Inc., Milford, Mass., USA) was used. A mobile phase of $\mathrm{MeCN} / 30 \mathrm{mmol} / 1 \mathrm{HFBA}$ (27: 73, volume/volume) was used. The flow rate was $1.0 \mathrm{ml} / \mathrm{min}$. The volume of each sample injected was $160 \mu$ l. For the detection of pentosidine, the fluorescence at $385 \mathrm{~nm}$ was measured on excitation at $335 \mathrm{~nm}$. The minimum amount of pentosidine detectable (signal-to-noise ratio, 2) was $1.6 \mathrm{pmol}$ per injection under our experimental conditions. The levels of pentosidine content in urine samples are expressed as micromoles of pentosidine per $1 \mathrm{~mol}$ of urinary creatinine.

\section{Gel filtration chromatography with Sephadex G-25}

Pentosidine in urine samples, hydrolysates of urine samples from healthy control subjects and diabetic patients or standard solution was fractionated by gel filtration chromatography on a Sephadex G-25 superfine (Pharmacia LKB Biotechnology AB) column $(1.2 \times 12.5 \mathrm{~cm})$. Two hundred microlitres of each sample which contained $25 \mathrm{mg}$ of $\mathrm{NaCl}$ was applied on the column equilibrated with $20 \mathrm{mmol} / \mathrm{l}$ phosphate buffer, $\mathrm{pH} 7.4$, containing $0.9 \% \mathrm{NaCl}$. Fractions of $0.92 \mathrm{ml}$ were collected. Blue dextran (Pharmacia LKB Biotechnology $\mathrm{AB}$ ) and $\mathrm{NaCl}$ were found to elute in fraction 5 and fractions $16-17$, respectively. The content of pentosidine in each fraction was assayed by fluorometry on HPLC as described above.
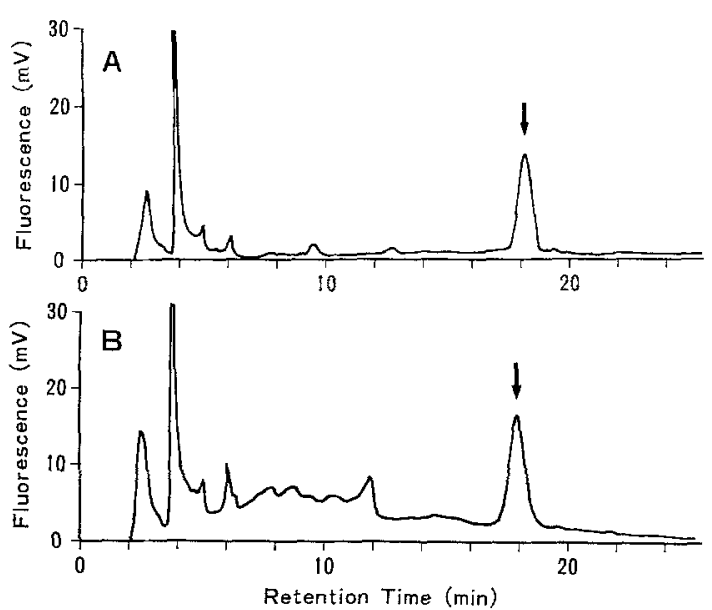

Fig. 1A, B. Chromatograms of SP-Sephadex C-25 elute: (A) urine, (B) hydrolysate of urine. Excitation/emission wavelength was $335 / 385 \mathrm{~nm}$. Arrow indicates elution position of authentic pentosidine

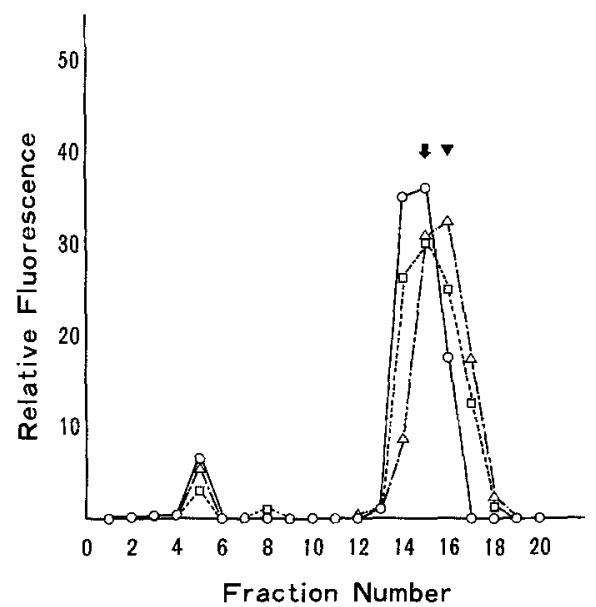

Fig.2. Chromatograms of urine on Sephadex G-25 superfine: control subjects, $-\mathrm{O}-$; diabetic patients, ---- $\square---;$; hydrolysates, $\cdots \Delta \cdots$. Excitation/emission wavelength was $335 / 385 \mathrm{~nm}$. Each point was calculated by the chromatography of three different samples. Arrow indicates elution position of authentic pentosidine. Arrowhead indicates elution position of $\mathrm{NaCl}$

\section{Preparation of standard solution}

Pentosidine was isolated from human articular cartilage. Methods of purification and characterization were shown in our previous paper [12].

\section{Urinary creatinine}

Before hydrolysis, urinary creatinine content was determined enzymatically on an aliquot of a urine sample using a Shimadzu CL-20 clinical chemistry analyser (Shimadzu, Kyoto, Japan).

\section{Urinary hydroxyproline}

The hydroxyproline content in hydrolysed samples was measured in a Model 835-50 automated amino acid analyser system (Hitachi, Tokyo, Japan). 
666

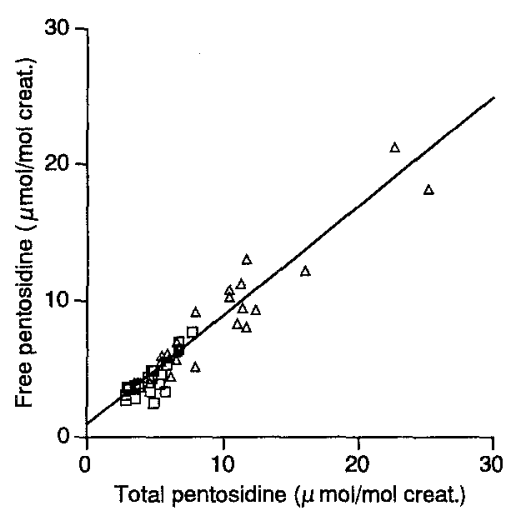

Fig. 3. Correlation between total and free pentosidine in urine from diabetic patients and control subjects. Diabetic patients, $\Delta ;$ control subjects, $\square$. Line indicates linear regression equation in the total of diabetic patients and control subjects: free form of pentosidine $=0.794 \times$ (total forms of pentosidine $)+1.186, r=0.938$, $p=0.0001$
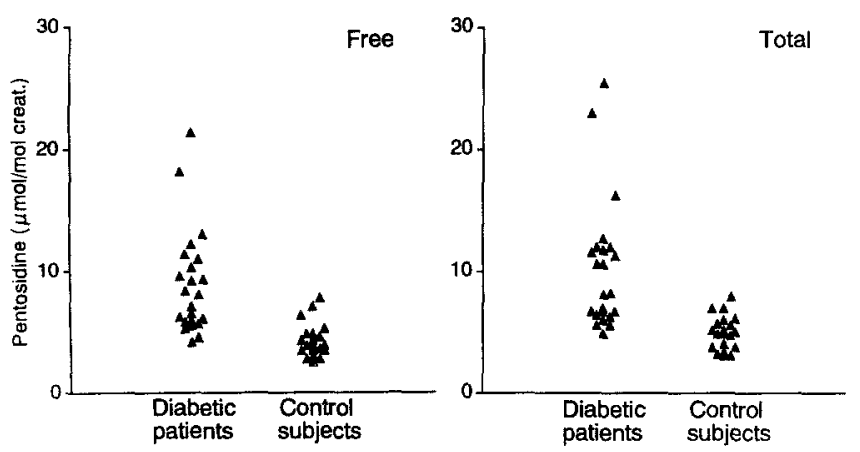

Fig.4. Pentosidine concentration in urine samples. Free, pentosidine in urine without hydrolysis; Total, pentosidine in hydrolysate of urine

\section{Statistical analysis}

Statistical analyses were performed with a StatView II program on a Macintosh computer. The statistical significance and correlation were determined with non-parametric statistics by Mann-Whitney $\mathrm{U}$ tests and the Spearmann rank correlation method, respectively. $P$ values below 0.05 were considered significant.

\section{Results}

Typical chromatograms of free form and total forms of pentosidine in urine from a 46-year-old diabetic male are shown in Figure 1. The chromatogram of free form (Fig. 1A) was simpler and clearer than that of total forms (Fig. 1B). This seems to suggest that some artifacts were produced during hydrolysis. Figure 2 shows typical gel-filtration chromatograms of pentosidine in urine samples, hydrolysates of urine samples or standard solution on a Sephadex G-25 superfine. Each point was obtained by the chromatography of three different samples. Since the arrow in the figure indicates the elution position of standard pentosidine, we conclude that most of the free form pentosidine has almost the same molecular weight as that of standard pentosidine. The recovery of standard pento-
M. Takahashi et al.: Urinary pentosidine from diabetic patients

sidine ( $n=6$, mean $\pm \mathrm{SD})$ and the coefficient of variation in the hydrolysate were $95.4 \pm 2.6$ and $2.9 \%$, respectively. In addition, since the level of free form pentosidine seemed to be the same as for the total forms of pentosidine, we compared values of two forms in each population of diabetic patients and control subjects and total populations of diabetic patients and control subjects. There was significant correlation between the values: free form pentosidine $(\mu \mathrm{mol} / \mathrm{mol}$ creatinine $)=0.753 \times($ total forms of pentosidine) $+1.186, r=0.938, p=0.0001$ in diabetic patients; free form pentosidine $=0.829 \times$ (total forms of pentosidine) $+0.2, r=0.820, p<0.02$ in control subjects; free form pentosidine $=0.794 \times$ (total forms of pentosidine) $+0.583, r=0.951, p=0.0001$ in the combined study populations (Fig. 3). The mean percentages ( \pm SD) of the free form per total forms were $89 \pm 15 \%$ (range $=64$ to $112 \%$ ) in diabetic patients; $88 \pm 16 \%$ (range $=48$ to $112 \%$ ) in control subjects; $89 \pm 15 \%$ in the total population.

Furthermore, we compared the values of the two forms per 1 mol of urinary creatinine between diabetic patients and control subjects. The mean value $( \pm S D)$ in diabetic patients was significantly higher than it in control subjects: $8.8 \pm 4.3 \mu \mathrm{mol} / \mathrm{mol}$ creatinine vs $4.2 \pm 1.4 \mu \mathrm{mol} / \mathrm{mol}$ creatinine, $p=0.0001$ for free form; $10.1 \pm 5.3 \mu \mathrm{mol} / \mathrm{mol}$ creatinine vs $4.7 \pm 1.4 \mu \mathrm{mol} / \mathrm{mol}$ creatinine, $p=0.0001$ for total forms (Fig. 4).

\section{Discussion}

During the first stage of the discovery of pentosidine, there was uncertainty as to whether it was formed artificially during acid hydrolysis [7]. Our results showed that more than $80 \%$ of pentosidine was present in free form and most of it remained intact after hydrolysis. These results support the idea that pentosidine is an endogenous component in humans.

Pentosidine is known to accumulate in human tissues with age and its accumulation accelerates in patients with diabetes and uraemia $[1,2,4,8-10]$. Although we first detected pentosidine in urine, the origin of pentosidine is unclear. Currently, there are two hypotheses: one is that pentosidine is formed with free forms of reducing sugars, arginine and lysine, but since it takes a long time for pentosidine formation reactions to occur under physiological conditions $[1,2,7]$, it seems unlikely that this mechanism would cause the production of pentosidine in vivo. The other hypothesis is that pentosidine is produced by degradation reactions of proteins in which pentosidine has accumulated. Hydroxyproline concentrations (mean $\pm \mathrm{SD}$ ) of hydrolysates of the urine samples were $5.93 \pm 2.22 \mathrm{mmol} / \mathrm{mol}$ creatinine in diabetic patients and $6.98 \pm 2.02 \mathrm{mmol} / \mathrm{mol}$ creatinine in control subjects. From these values, the concentrations of pentosidine in human urine were about $1.63 \mathrm{nmol} / \mathrm{mg}$ collagen in diabetic patients and about $0.64 \mathrm{nmol} / \mathrm{mg}$ collagen in control subjects, and the urinary excretion of pentosidine was approximately $75 \mu \mathrm{g}$ per day in diabetic patients and $29 \mu \mathrm{g}$ per day in the control subjects. The concentrations of pentosidine per mg collagen shown in this report are the 
highest of all results previously reported [4, 5, 7-9], which suggests that there are some mechanisms in tissues which prevent pentosidine from accumulating. We currently have no data which identifies the cause of increased concentration of pentosidine in the urine of diabetic patients; however, it is possible that oxidative stress may play an important role [6].

In this study, using cation exchange chromatography (SP-Sephadex C-25), we have shown that more than $80 \%$ of urinary pentosidine is present in its free form. We also provide the evidence that the levels of urinary pentosidine in patients with diabetes were significantly higher than those in control subjects. Pentosidine is a fluorescent compound, and using fluorometry with HPLC, we could measure pentosidine in urine at a concentration as low as 1.6 pmol per injection. Therefore, these results suggest that the free form of pentosidine in human urine could be a biomarker for diagnostic use and will be useful in the management of diabetic patients. Our results also reveal that it may be possible to develop a new method for detection of the levels of urinary pentosidine using immunological techniques.

Acknowledgements. We wish to thank Professor Y.Fujise for encouragement.

\section{References}

1. Monnier VM (1990) Nonenzymatic glycosylation, the Maillard reaction and the aging process. J Gerontol 45: B105-B111

2. Dyer DG, Blackledge JA, Katz BM et al. (1991) The Maillard reaction in vivo. $Z$ Ernährungswiss $30: 29-45$

3. Bunn HF (1981) Evaluation of glycosylated hemoglobin in diabetic patients. Diabetes 30: 613-617
4. Lyons TJ, Silvestri G, Dunn JA, Dyer DG, Baynes JW (1991) Role of glycation in modification of lens crystallins in diabetic senile cataracts. Diabetes 40: 1010-1015

5. Lyons TJ, Bailie KE, Dyer DG, Dunn JA, Baynes JW (1991) Decrease in skin collagen glycation with improved glycemic control in patients with insulin-dependent diabetes mellitus. J Clin Invest $87: 1910-1915$

6. Baynes JW (1991) Role of oxidative stress in development of complications in diabetes. Diabetes 40: 405-412

7. Sell DR, Monnier VM (1989) Structure elucidation of a senescence cross-link from human extracellular matrix. Implication of pentoses in the aging process. J Biol Chem 264: 21597-21602

8. Odetti P, Fogarty J, Sell DR, Monnier VM (1992) Chromatographic quantitation of plasma and erythrocyte pentosidine in diabetic and uremic subjects. Diabetes 41: 153-159

9. Sell DR, Monnier VM (1990) End-stage renal disease and diabetes catalyze the formation of a pentose-derived crosslink from aging human collagen. J Clin Invest 85: 380-384

10. Sell DR, Nagaraj RH, Grandhee SK et al. (1991) Pentosidine: a molecular marker for the cumulative damage to proteins in diabetes, aging, and uremia. Diabetes Metab Rev 7: 239-251

11. Takahashi M, Ohisi T, Aoshima H, Kushida K, Inoue T, Horiuchi K (1993) Pre-fractionation with cation exchanger for determination of intermolecular crosslinks, pyridinoline and pentosidine, in hydrolysate. J Liq Chromatogr 16: 1355-1370

12. Uchiyama A, Ohishi T, Takahashi M et al. (1991) Fluorophores from aging human articular cartilage. J Biochem 110: 714-718

Received: 10 November 1992

and in revised form: 16 March 1993

Dr. M. Takahashi

Department of Orthopaedic Surgery

Hamamatsu University School of Medicine

3600 Handa, Hamamatsu

Shizuoka 431-31

Japan 